\title{
Identification and signatures based on NP-hard problems of indefinite quadratic forms
}

\author{
Rupert J. Hartung and Claus-Peter Schnorr \\ Communicated by
}

\begin{abstract}
We prove NP-hardness of equivalence and representation problems of quadratic forms under probabilistic reductions, in particular for indefinite, ternary quadratic forms with integer coefficients. We present identifications and signatures based on these hard problems. The bit complexity of signature generation and verification is quadratic using integers of bit length 150 .
\end{abstract}

Keywords. Identification, signature, indefinite quadratic forms, NP-hardness, proof of knowledge, anisotropic form, equivalence class.

AMS classification. 11E20, 94A60, 68D15, 11D09, 11R29.

\section{Introduction}

The arithmetic theory of quadratic forms goes back to FERMAT, LEGENDRE, LAGRANGE, and GAUSS. Algorithmic problems for lattices and quadratic forms have been promoted by the LLL-algorithm [14]. Recently definite forms or lattices gave rise to cryptographic protocols related to the NP-hard problems of finding a shortest or a closest lattice vector; see [15], [20] for hardness results and [1], [8], [11], [12] for applications. Cryptographic protocols based on NP-hard problems seem to withstand attacks by quantum computers. However, lattice cryptography requires lattices of high dimension. This yields long cryptographic keys and slow protocols.

By contrast, we present identification and signatures based on hard problems of quadratic forms in dimension three and four. Bounded solutions of equivalence and representation problems for indefinite ternary forms are shown to be NP-hard for probabilistic reductions. This follows from ADLEMAN and MANDERS [18] who proved NP-completeness of deciding solvability of inhomogeneous binary quadratic equations over the integers. Signature generation and verification have quadratic bit complexity for integers of bit length 150 . Note that RSA has cubic bit complexity for much longer integers.

Outline. Section 2 introduces computational problems of quadratic forms. Section 3 presents an identification scheme that proves knowledge of an equivalence transform for ternary anisotropic forms. It performs one LLL-reduction and a few arithmetic steps per round. Section 4 extends the identification to long challenges and signatures. Section 5 gives NP-hardness proofs. Section 6 presents polynomial time solutions for problems of isotropic forms of odd squarefree determinant. Section 7 characterizes anisotropic forms. 


\section{The equivalence problem of quadratic forms}

Quadratic forms. An $n$-ary quadratic form (or simply form) $f$ over $\mathbb{Z}$ is a homogeneous quadratic polynomial $f=f_{A}=\sum_{i, j=1}^{n} a_{i, j} x_{i} x_{j}$ with coefficients $a_{i, j}=a_{j, i} \in$ $\frac{1}{2} \mathbb{Z}$ for $i \neq j, a_{i, i} \in \mathbb{Z}, A=\left(a_{i, j}\right) \in \mathbb{Z}^{n \times n}$. Note that $f$ takes integer values for $x_{1}, \ldots, x_{n} \in \mathbb{Z}$. By definition $\operatorname{det} f=\operatorname{det} A, \operatorname{dim} f=n$.

Equivalence classes. Let $f=f_{A}$ be an $n$-ary form. For $T \in \mathbb{Z}^{n \times n}$ let $f_{A} T$ denote the form $f_{T^{t}} A T$. The forms $f, f T$ are called equivalent if $T \in \mathrm{GL}_{n}(\mathbb{Z})$, i.e., $|\operatorname{det} T|=$ 1. Obviously $\operatorname{det}(f T)=(\operatorname{det} T)^{2} \operatorname{det} f=\operatorname{det} f$. We call the equivalence class of $f$ simply the class of $f$. Let $\mathcal{O}(f)=\{T: f T=f,|\operatorname{det} T|=1\}$ denote the group of automorphisms of $f$. The textbook by CASSELS [3] surveys of the structure of classes.

Relevant properties of forms are: $f$ is regular if det $f \neq 0 ; f$ is indefinite if $f$ takes both positive and negative values. Otherwise $f$ is either positive or negative, positive forms correspond to the Gram matrices $A=B^{t} B \in \mathbb{R}^{n \times n}$ of lattice bases $B \in \mathbb{R}^{n \times n}$. $f=f_{A}$ is primitive if $\operatorname{gcd}\left(a_{i j} \mid 1 \leq i, j \leq n\right)=1 ; f$ is isotropic if $f(\mathbf{u})=0$ holds for some nonzero $\mathbf{u} \in \mathbb{Z}^{n}$, otherwise $f$ is anisotropic. Every regular isotropic form is necessarily indefinite. The forms $f=\sum_{i=1}^{n} a_{i} x_{i}^{2}$ are called diagonal.

We study the equivalence problem of forms $f \in \mathcal{Q}$ for various sets of forms $\mathcal{Q}$.

\section{Computational equivalence problem, $\operatorname{CEP}(\mathcal{Q})$}

GIVEN: equivalent forms $f, g \in \mathcal{Q}$.

FIND: $\quad T \in \mathrm{GL}_{n}(\mathbb{Z})$ such that $g=f T$.

Representations. An $n$-ary form $f$ represents the integer $m$ if there exists $\mathbf{u} \in \mathbb{Z}^{n} \backslash\{0\}$ such that $f(\mathbf{u})=m$. The representation $\mathbf{u}$ is primitive if $\operatorname{gcd}\left(u_{1}, \ldots, u_{n}\right)=1$. Let $\mathbf{C R}(\mathcal{Q})$ denote the problem to find for given $f \in \mathcal{Q}$ and $m \in \mathbb{Z}$ a primitive $\mathbf{u} \in \mathbb{Z}^{n}$ such that $f(\mathbf{u})=m$ whenever such $\mathbf{u}$ exists.

We show in section 5 that bounded solutions of CEP and CR are NP-hard to find for indefinite forms $f$ of $\operatorname{dim} f=3$ and for definite forms $f$ of $\operatorname{dim} f=5$.

LLL-reduction [14] extends from lattice bases and definite forms to indefinite forms. LLL-forms $f_{A}, A=\left(a_{i, j}\right)$ satisfy $a_{1,1}^{2} \leq 2^{n / 2}(\operatorname{det} A)^{2 / n}$. There is a poly-time algorithm that transforms $f$ into an LLL-form $f T$ with $T \in \mathrm{GL}_{n}(\mathbb{Z})$, see [13], [25], [27].

\section{Identification based on the equivalence problem}

We present efficient proofs of knowledge of an equivalence transform for indefinite forms. We use anisotropic forms for keys because there exist poly-time (unbounded) solutions of CEP and CR for isotropic forms $f$ given the factorization of $\operatorname{det} f$, see section 6. No relevant poly-time solutions are known for CEP and CR and anisotropic forms. Let $k \in \mathbb{N}$ be a security parameter.

Key generation. Pick an anisotropic form $f_{1}=a x^{2}+b y^{2}-c z^{2}$ with $a, b, c \in_{R}\left[1,2^{k}[\right.$. Using the factorization of $\operatorname{det} f$ verify that $f$ is anisotropic, see section 7 .

Generate $T$ and $f_{1} T$ by $\mathbf{C T}\left(f_{1}\right)$ and set $S:=T, f_{0}:=f_{1} S$.

The public key is $f_{0}, f_{1}$, the private key is $S$. 
The goal of the following procedure $\mathbf{C T}(f)$ is to generate within $(\mathcal{P}, \mathcal{V})_{1}$ forms $f_{b} S^{b} T$ for $b=0,1$ via $\mathbf{C T}\left(f_{b} S^{b}\right)$ with (practically) indistinguishable distributions.

$\mathbf{C T}(f)$ : Computes a randomized $T=\left(t_{i, j}\right) \in \mathrm{GL}_{3}(\mathbb{Z})$ and an LLL-form $f:=f T$.

1. Pick $\left.t_{i, j} \in_{R}\right]-2^{k}, 2^{k}$ [at random for $j \neq 1$. Compute the $t_{i, 1}^{a d j}$ of $\left(t_{i, j}^{a d j}\right)=T^{-1} \operatorname{det} T$ : $\left(t_{1,1}^{a d j}, t_{2,1}^{a d j}, t_{3,1}^{a d j}\right):=\left(t_{2,2} t_{3,3}-t_{2,3} t_{3,2}, t_{1,2} t_{3,3}-t_{1,3} t_{3,2}, t_{1,2} t_{3,3}-t_{1,3} t_{3,2}\right)$.

2. Compute the $t_{i, 1}$ by solving $\sum_{i=1}^{3} t_{i, 1} t_{i, 1}^{a d j}=\operatorname{gcd}\left(t_{1,1}^{a d j}, t_{2,1}^{a d j}, t_{3,1}^{a d j}\right)=: \operatorname{gcd}$ for $t_{i, 1} \in \mathbb{Z}$ by the extended gcd-algorithm. If gcd $\neq 1$ then repeat with some new $t_{i, j}, j \neq 1$.

3. LLL-reduce $f T$ to $f T T^{\prime}$, replace $T:=T T^{\prime}$, and denote $\mathbf{C T}(f):=f T$.

Step 3 balances the initially large $\left|t_{i, 1}\right| \leq \max _{i}\left|t_{i, 1}^{a d j}\right|<2^{2 k+2}$ with the smaller $t_{i, j}$, $j \neq 1$. The random initial $t_{i, j}, j \neq 1$ randomize the leading and the least significant $k$ bits of the final $t_{i, j}$.

Identification $(\mathcal{P}, \mathcal{V})_{1}$

The prover $\mathcal{P}$ proves to the verifier $\mathcal{V}$ knowledge of $S$.

1. $\mathcal{P}$ sends as commitment the LLL-form $\bar{f}:=f_{0} T:=\mathbf{C T}\left(f_{0}\right)$,

2. $\mathcal{V}$ sends a random one-bit challenge $b \in_{R}\{0,1\}$,

3. $\mathcal{P}$ sends the reply $R_{b}:=S^{b} T$, and $\mathcal{V}$ checks that $f_{b} R_{b}=\bar{f}$ and $\left|\operatorname{det} R_{b}\right|=1$.

Obviously, the honest prover $\mathcal{P}$ withstands the test $f_{b} R_{b}=\bar{f}$.

Proof of knowledge. Consider a fraudulent $\widetilde{\mathcal{P}}$ that sends arbitrary $\bar{f}, \bar{R}_{b}$. The trivial $\widetilde{\mathcal{P}}$ guesses $b$ in step 1 with probability $\frac{1}{2}$, sends the LLL-form $\bar{f}:=f_{b} T:=\mathbf{C T}\left(f_{b}\right)$ and replies $\bar{R}_{b}:=T$. Then $\widetilde{\mathcal{P}}$ withstands the verification with probability $\frac{1}{2}$. The probability $\frac{1}{2}$ cannot be increased. If an arbitrary $\widetilde{P}$ withstands the verification for the same $\bar{f}$ and both challenges $b=0,1$ then $f_{0} \bar{R}_{0}=\bar{f}=f_{1} \bar{R}_{1}$, and thus $f_{1} \bar{R}_{1} \bar{R}_{0}^{-1}=f_{0}$. This yields an alternative private key $\bar{S}=\bar{R}_{1} \bar{R}_{0}^{-1} \in \mathcal{O}\left(f_{1}\right) S$ in time proportional to $\left|(\widetilde{\mathcal{P}}, \mathcal{V})_{1}\right|\left(\right.$ which denotes the number of steps of $\left.(\widetilde{\mathcal{P}}, \mathcal{V})_{1}\right)$ :

Theorem 3.1. An arbitrary fraudulent prover $\widetilde{P}$ that succeeds in $(\widetilde{\mathcal{P}}, \mathcal{V})_{1}$ with probability $\varepsilon>\frac{1}{2}$ finds some $\bar{S} \in \mathcal{O}\left(f_{1}\right) S$ in expected time $\left|(\widetilde{\mathcal{P}}, \mathcal{V})_{1}\right| /\left(\varepsilon-\frac{1}{2}\right)$.

Secret key protection. We give heuristic arguments that the protocol $(\mathcal{P}, \mathcal{V})_{1}$ is secure against a fraudulent verifier $\widetilde{\mathcal{V}}$, in particular that the information $R_{b}$ released to $\widetilde{\mathcal{V}}$ does not help to compute some $\bar{S} \in \mathcal{O}\left(f_{1}\right) S$.

Only P's reply $R_{1}=S T$ depends on $S$ whereas $R_{0}=T$ is independent of $S$. However, $f_{1}$ and $\bar{f}$ define $R_{1}$ uniquely up to automorphisms of $f_{1}$ by the equation $f_{1} R_{1}=\bar{f}$. Note that $f_{1}$ and $\bar{f}$ should be nearly independent of $S$ since $f_{0}=f_{1} S$ and $\bar{f}=f_{1} S T$ are generated by $\mathbf{C T}$ using independent random bits. Therefore, nearly all information that $S T$ contains about $S$ should depend on the choice of automorphisms of $f_{1}$, and these automorphisms must be sufficiently small. There provably exist polynomial size $A \in \mathcal{O}\left(f_{1}\right)$ [6] but their known bounds are extremely large having polynomial degree larger than 500 .

The protocol $(\mathcal{P}, \mathcal{V})_{1}$ would be statistical zeroknowledge if the distributions of $T$ 
and $S T$ differ negligibly. We did not yet check this out experimentally.

For further protection we can let $\mathcal{P}$ randomize $R_{1}=A_{1} S T$ by chosing $A_{1} \in \mathcal{O}\left(f_{1}\right)$ according to some random distribution; to this end it suffices to precompute generators of $\mathcal{O}\left(f_{1}\right)$ once and for all. If the resulting distributions of $R_{1}$ are close for varying $S T$, the $R_{1}$ will practically only depend on $\bar{f}$ and $f_{1}$. We conjecture that this additional measure of protection is not necessary:

Conjecture. $\mathcal{P}$ does not reveal relevant information about $S$.

This extends to independent sequential executions of $(\mathcal{P}, \mathcal{V})_{1}$, since $\mathcal{P}$ generates the corresponding $\bar{f}:=f_{0} T:=\mathbf{C T}\left(f_{0}\right)$ using independent random bits. Since $(\mathcal{P}, \mathcal{V})_{1}$ is restricted to one-bit challenges, a security level $2^{100}$ requires 100 independent executions of $(\mathcal{P}, \mathcal{V})_{1}$ and 300 rounds.

\section{Three round identification and signatures}

Our signatures correspond to identification in three rounds with long challenges, where the verifier is simulated using a hash function. This identification releases some information about the private key which we argue to be irrelevant. This information cannot be combined over several identifications, but it reduces CEP to $(n-1)$-ary subforms of $f_{0}, f_{1}$. To make the reduced CEP hard we increase $\operatorname{dim} f_{1}$ to $n=4$.

Private key $S \in \mathrm{GL}_{4}(\mathbb{Z})$,

Public key anisotropic forms $f_{0}=f_{1} S, f_{1}=\sum_{i=1}^{4} a_{i} x_{i}^{2}$ with $(-1)^{i} a_{i} \in\left[1,2^{k}[\right.$.

Key generation. Apply a public hash function to a public / private random 100-bit seed to generate the pseudo-random bits for the construction of $S$ via $\mathbf{C T}\left(f_{1}\right)$ and the pseudo-random entries $\left|a_{i}\right| \in\left[1,2^{k}\right.$ of $f_{1}$ (make sure that $p^{2 k} \mid d, \quad p^{2 k+1} \nmid d$ holds for a small prime $p$ and $d:=a_{1} a_{2} a_{3} a_{4}$ and that $\prod_{i<j}\left(a_{i}, a_{j}\right)_{p}=(-1)^{p \bmod 2}$ holds as required by Theorem 7.2.). This way the public / private keys have bit lengths $10 k+100$ and 100 , respectively. Note that CT, extended to $n=4$ performs arithmetic steps on $3 k$-bit integers. The forms $f_{0}, f_{1}$ are indefinite and so are all ternary subforms of $f_{0}, f_{1}$.

\section{Three round identification $(\mathcal{P}, \mathcal{V})_{2}$}

Prover $\mathcal{P}$ solves problems posed by the verifier $\mathcal{V}$ that are hard without knowledge of $S$

1. $\mathcal{P}$ sends the LLL-form $\bar{f}:=f_{0} \bar{T}:=\mathbf{C T}\left(f_{0}\right)$ to $\mathcal{V}$,

2. $\mathcal{V}$ computes the LLL-form $f^{\prime}:=\bar{f} T^{\prime}:=\mathbf{C T}(\bar{f})$ and sends $T^{\prime}$ to $\mathcal{P}$,

3. $\mathcal{P}$ sends $\mathbf{e}_{b}^{\prime}:=S^{b} \bar{T} T^{\prime} \mathbf{e}_{b}$ for $b=0,1$ and $\mathbf{e}_{1}:=(1,0,0,0)^{t}, \mathbf{e}_{0}:=(0,0,0,1)^{t}$,

$\mathcal{V}$ checks that $f_{b} \mathbf{e}_{b}^{\prime}=\bar{f} T^{\prime} \mathbf{e}_{b}$ for $b=0,1 . \quad$ (we abbreviate $T:=\bar{T} T^{\prime}$ )

Note that $\mathcal{P}$ randomizes $\bar{T}$ to protect against a fraudulent $\widetilde{\mathcal{V}}$ and $\mathcal{V}$ randomizes $T^{\prime}$ against a fraudulent $\widetilde{\mathcal{P}}$. Importantly $\widetilde{\mathcal{V}}$ does not know $T=\bar{T} T^{\prime}$ but only $T^{\prime}$.

Security against a fraudulent $\widetilde{\mathcal{P}}$. A successful $\widetilde{\mathcal{P}}$ must find solutions $\mathbf{e}_{b}^{\prime}$ of $f_{b}\left(\mathbf{e}_{b}^{\prime}\right)=m$ for both $b=0,1$, where the randomized $m:=\bar{f} T^{\prime} \mathbf{e}_{b}$ is chosen by the verifier. The 
problem to find a small solution $\mathbf{e}_{b}^{\prime}$ without the private key is NP-hard by Theorem 5.1. The fastest known method for solving $f_{b} \mathbf{e}_{b}^{\prime}=\bar{f} T^{\prime} \mathbf{e}_{b}$ for $\mathbf{e}_{b}^{\prime}$ is by reconstructing $S$.

By revealing $\mathbf{e}_{1}^{\prime}=\left(\sum_{i=1}^{4} s_{j, i} t_{i, 1} \mid j=1, \ldots, 4\right)^{t}$ the honest $\mathcal{P}$ proves bounds on $\sum_{i}\left|s_{j, i}\right|$ for $j=1, \ldots, 4, S=\left(s_{i, j}\right)$. $\mathcal{P}$ comes close to prove a bound on $\left|s_{1,1}\right|$ as is required for the NP-hardness results of section 5 .

Security against a fraudulent $\tilde{\mathcal{V}}$. Note that $\mathcal{P}$ reveals $S$ if $\mathcal{P}$ replies to various challenges $T^{\prime}$ for the same $\bar{f}$, it suffices that $\widetilde{\mathcal{V}}$ generates challenges by cyclically permuting the columns of some $T^{\prime}$. Randomization of $\bar{f}$ via $\mathbf{C T}$ protects against this attack.

The novelty compared to $(\mathcal{P}, \mathcal{V})_{1}$ is that step 3 sends information about $S^{b} \bar{T} T^{\prime}$ for both $b=0,1$ by sending $\mathbf{e}_{b}^{\prime}=S^{b} \bar{T} T^{\prime} \mathbf{e}_{b}$ for $b=0,1 . S^{b} \bar{T} T^{\prime}$ releases for a single $b$ no relevant information about $S$ as has been explained in section 3 .

Next, we consider the following attacks that combine both replies $\mathbf{e}_{b}^{\prime}$ :

1. recovering direct information about $S$ from $\mathbf{e}_{0}^{\prime}, \mathbf{e}_{1}^{\prime}$,

2. reconstruct $S T$ from $\mathbf{e}_{1}^{\prime}$ and $T$ from $\mathbf{e}_{0}^{\prime}$. This would reveal $S$.

1. The small, orthogonal vectors $\mathbf{e}_{0}, \mathbf{e}_{1}$ are chosen so that $\bar{T} T^{\prime} \mathbf{e}_{b}$ for $b=0,1$ are nearly statistically independent for random $T=\bar{T} T^{\prime}$. This protects against the following attack. If $\mathbf{e}_{1} \in \operatorname{span}\left(\mathbf{e}_{0}\right)$ then $T \mathbf{e}_{1} \in \operatorname{span}\left(T \mathbf{e}_{0}\right)$ and thus $\mathbf{e}_{1}^{\prime} \in \operatorname{span}\left(S \mathbf{e}_{0}^{\prime}\right)$. This would completely disclose $S$ by a few identifications. This attack can be extended to the case that $\mathbf{e}_{0}, \mathbf{e}_{1}$ are nearly parallel.

It is crucial that $T \mathbf{e}_{0}, T \mathbf{e}_{1}$ are for $T=\bar{T} T^{\prime}$ nearly statistically independent (which holds for small, orthogonal $\mathbf{e}_{0}, \mathbf{e}_{1}$ ). This makes the information released by the combination of $\mathbf{e}_{0}^{\prime}, \mathbf{e}_{1}^{\prime}$ to $\widetilde{\mathcal{V}}$ practically negligible, and completely foils the described attack. Moreover, the released information cannot be combined for several identifications as the $T$ are generated by independent random bits.

2. Hardness of recovering ST from $\mathbf{e}_{1}^{\prime}$. Let $f_{1}=\sum_{i=1}^{4} a_{i} x_{i}^{2}=f_{A_{1}}, f^{\prime}=f_{A^{\prime}}=f_{1} S T$. Given $\mathbf{e}_{1}^{\prime}=S T \mathbf{e}_{1}$ we can transform $f^{\prime}$ into $f^{\prime \prime}=f_{A^{\prime \prime}}:=f^{\prime} T^{\prime \prime}$ such that $a_{1}=a_{1,1}^{\prime \prime}$, and thus

$$
\left(\begin{array}{cc}
1 & \mathbf{0}^{t} \\
\mathbf{u} & Q^{t}
\end{array}\right) A_{1}\left(\begin{array}{cc}
1 & \mathbf{u}^{t} \\
\mathbf{0} & Q
\end{array}\right)=A^{\prime \prime}=:\left(\begin{array}{cc}
a_{1} & a_{1} \mathbf{u}^{t} \\
a_{1} \mathbf{u} & A_{+}^{\prime \prime}
\end{array}\right)
$$

holds for some $\mathbf{u} \in \mathbb{Z}^{3}, Q \in \mathrm{GL}_{3}(\mathbb{Z}), A_{+}^{\prime \prime} \in \mathbb{Z}^{3 \times 3}$. We rewrite (5) as

$$
Q^{t} A_{+} Q=A_{\mathbf{u}}^{\prime \prime} \text { for } A_{\mathbf{u}}^{\prime \prime}:=A_{+}^{\prime \prime}+a_{1} \mathbf{u u}^{t},
$$

where $A_{+}$is the diagonal submatrix of $A_{1}$ with diagonal $\left(a_{2}, a_{3}, a_{4}\right)$. Solving equation (5) for $Q \in \mathrm{GL}_{3}(\mathbb{Z})$ requires an unbounded solution of CEP for $f_{A_{+}}, f_{A_{\mathrm{u}}^{\prime \prime}}$. Thus, releasing $\mathbf{e}_{1}^{\prime}$ reduces CEP to ternary anisotropic forms and the recovering of $\mathbf{u}$.

Hardness of recovering $\mathbf{u}$. Equation (5) implies that $\operatorname{det} A_{+}^{\prime \prime}=\operatorname{det} A_{\mathbf{u}}^{\prime \prime}$, and this equation can be written as $\operatorname{det} A_{+}^{\prime \prime}=f_{D}(\mathbf{u})+a_{2} a_{3} a_{4}$ for the indefinite, diagonal matrix $D$ with diagonal $a_{1}\left(a_{3} a_{4}, a_{2} a_{4}, a_{2} a_{3}\right)$ and $a_{1}, a_{3}<0<a_{2}, a_{4}$. Hence the construction of $\mathbf{u}$ from $\mathbf{e}_{1}^{\prime}$ requires to solve $f_{D}(\mathbf{u})=\operatorname{det} A_{+}^{\prime \prime}-a_{2} a_{3} a_{4}$. This problem can be made hard by ensuring that $f_{D}$ is anisotropic, see sections 6,7 . Then no isotropic vector can help 
to recover $\mathbf{u}$.

The best known attack. In order to solve CEP for $f=f_{A_{+}}$and $f^{\prime}=f_{A_{\mathrm{u}}^{\prime \prime}}$ it is promising to exhaust the reduced forms $f_{A}$ in the class of $f, f^{\prime}$ by simple transforms of $\mathrm{GL}_{3}(\mathbb{Z})$ : permute two rows and columns of $A$ and transform the result into an reduced form. For $k$-bit coefficients of $f, f^{\prime}$ we have that $d:=\operatorname{det} f=O\left(2^{3 k}\right)$ and the number of on average there are $\Theta(d)$ reduced forms in the class of $f$. ( A simple counting argument shows that there are $\Theta\left(2^{6 k}\right)$ ternary, indefinite forms with coefficients of size $O\left(2^{k}\right)$ that are reduced in the sense of GAUSS [7, art. 272], see also LAGARIAS [16, sect. 4 D]. These distribute over $\Theta\left(2^{3 k}\right)$ determinants $d$ which gives on average $\Theta\left(2^{3 k}\right)$ reduced forms per $d$. ) This can solve CEP for $f, f^{\prime}$ in average time $O\left(2^{3 k}\right)$.

Conclusion. The problem to recover $Q, \mathbf{u}$ and thus $S T$ from $\mathbf{e}_{1}^{\prime}=S T \mathbf{e}_{1}$ requires $\Omega\left(2^{3 k}\right)$ arithmetic steps for all known algorithms. We are not aware of any better method for solving $f_{b} \mathbf{e}_{b}^{\prime}=\bar{f} T^{\prime} \mathbf{e}_{b}$ for $\mathbf{e}_{b}^{\prime}$. Therefore, $k=50$ seems to provide sufficient security.

Most instances of the recovering problem are subexponential for $n=3$. For indefinite, binary $A_{+}, D$, the multiplicative structure of the Gaussian cycle [7, art.183ff] of reduced forms containing $f_{A_{+}}, f_{D}$, yields subexponential algorithms see [2, chap. 11].

Signatures. Extend the keys of $(\mathcal{P}, \mathcal{V})_{2}$ by random seeds $s^{\prime}, s^{*} \in_{R}\{0,1\}^{100}, s^{\prime}$ public and $s^{*}$ private. To generate a signature for message $m$ follow $(\mathcal{P}, \mathcal{V})_{2}$ but simulate $\mathcal{V}$ randomized by $H\left(m, s^{\prime}\right)$ for a public hash function $H$.

Signature generation. Compute $\bar{T}, \bar{f}:=f_{0} \bar{T}$ by $\mathbf{C T}\left(f_{0}\right)$ randomized by $H\left(m, s^{*}\right)$, keep $\bar{T}$ secret. Compute $\bar{f} T^{\prime}, T^{\prime}$ by $\mathbf{C T}(\bar{f})$ randomized by $H\left(m, s^{\prime}\right)$, set $\mathbf{e}_{b}^{\prime}:=S^{b} \bar{T} T^{\prime} \mathbf{e}_{b}$. The resulting signature $\left(\bar{f}, \mathbf{e}_{0}^{\prime}, \mathbf{e}_{1}^{\prime}\right)$ consists of $18 k$-bit integers, the entries of $\mathbf{e}_{0}^{\prime}, \mathbf{e}_{1}^{\prime}, \bar{f}$.

Verification computes $T^{\prime}$ from $m, s^{\prime}$ and checks that $f_{b} \mathbf{e}_{b}^{\prime}=\bar{f} T^{\prime} \mathbf{e}_{b}$ for $b=0,1$.

The bit complexity of signature generation and verification is quadratic $O\left(k^{2}\right)$, its main work is LLL-reduction within CT. LLL-reduction using orthogonalisation in floating point arithmetic has quadratic bit complexity using school multiplication [21], [25].

\section{NP-hardness}

5.1 The results. Recall that a set $S \subset \mathbb{Z}^{*}$, consisting of sequences of integers, is in NP if $S$ is decidable in nondeterministic poly-time. We prove probabilistic NP-hardness of decisional variants of $\mathbf{C E P}(\mathcal{Q})$ and $\mathbf{C R}(\mathcal{Q})$ that ask for a solution $\mathbf{x}$ with a given bound. We first present the results and thereafter the proofs.

\section{Decisional Bounded Representation Problem, $\operatorname{DBR}(\mathcal{Q})$}

GIVEN: $f \in \mathcal{Q}, m \in \mathbb{N}, c \in \mathbb{N}$, the factorization of $\operatorname{det} f$.

$D E C I D E$ : whether $\exists$ primitive $\mathbf{x} \in \mathbb{Z}^{n}: f(\mathbf{x})=m$ and $\left|x_{1}\right| \leq c$.

The given factorization of det $f$ does not decrease the worst-case complexity.

Recall that the class RP of random polynomial time consists of all sets $S \subset \mathbb{Z}^{*}$ for which there is a probabilistic, poly-time algorithm which accepts every $s \in S$ with 
probability $\geq \frac{1}{2}$ and rejects every $s \in \mathbb{Z}^{*} \backslash S$ with probability 1 .

Let $\mathcal{Q}_{\text {ind }}$ consist of all indefinite, primitive forms $f$ of $\operatorname{dim} f=3$.

Theorem 5.1. $\mathbf{D B R}\left(\mathcal{Q}_{\text {ind }}\right)$ is probabilistic $\mathbf{N P}$-hard, i.e., $\mathbf{N P} \subseteq \mathbf{R} \mathbf{P}^{\mathbf{D B R}\left(\mathcal{Q}_{\text {ind }}\right)}$.

Therefore, if $\mathbf{D B R}\left(\mathcal{Q}_{i n d}\right)$ is in $\mathbf{R P}$ then so is every $\mathbf{N P}$-set. The proof of Theorem 5.1 reduces a Boolean form $\Phi$ in 3 -CNF to a form $f=2 x^{2}+2 b y z$, where $\frac{1}{2} \operatorname{det} f \in \mathbb{Z}$ is odd. Theorem 5.1 follows essentially from [18].

Dimension 3 is minimal for this hardness result. In fact, an algorithm of Gauß computes small representations for binary forms in subexponential time if the class number is small (see [7, art. 183-221], [5, sec. 5.2 and 5.6]).

For isotropic ternary forms of odd squarefree determinant unbounded solutions of CEP and CR can be found in poly-time given an isotropic vector. For anisotropic forms, by contrast, CEP, CR seem to be hard on average.

Theorem 5.2 shows that deciding whether a lattice of dimension 5 has a vector of given length is NP-hard.

Theorem 5.2. Let $\mathcal{Q}_{d f}$ consist of all positive definite forms $f$ of $\operatorname{dim} f=5$. Then $\operatorname{DBR}\left(\mathcal{Q}_{d f}\right)$ is NP-complete.

Representation problems for definite forms are by Theorem 5.2 harder than equivalence problems. In fact, equivalence transformations of definite forms can be efficiently computed in constant dimension by computing shortest lattice vectors [22], [23].

Incomplete forms. If some coefficients $a_{i, j}$ of $A$ are undefined, $a_{i, j}=*$, and if $\operatorname{det} A$ does not depend on the undefined $a_{i, j}$ then we call $f_{A}$ incomplete. For example the form $f=a x^{2}+b x y+2 a_{1,3} x z+z^{2}$ is incomplete for $a_{1,3}=*$. A completion of $f_{A}$ defines the undefined $a_{i, j}$ of $A$.

\section{Decisional Bounded Equivalence Problem, $\operatorname{DBE}(\mathcal{Q})$}

GIVEN: $\quad f, g \in \mathcal{Q}, c \in \mathbb{N}$, the factorization of $\operatorname{det} f$.

$D E C I D E$ : whether $\exists T \in \mathrm{GL}_{n}(\mathbb{Z}): f T=g,\left|t_{1,1}\right| \leq c$.

$\operatorname{DBE}(\mathcal{Q})$ for given $n$-ary incomplete forms $f, g \in \mathcal{Q}$ asks for the existence of $T \in$ $\mathrm{GL}_{n}(\mathbb{Z})$ and completions $\bar{f}, \bar{g}$ of $f, g$ such that $\bar{f} T=\bar{g},\left|T_{1,1}\right| \leq c$.

Let $\mathcal{Q}_{\text {inin }}$ extend the set $\mathcal{Q}_{\text {ind }}$ by all incomplete, indefinite forms $f$ of $\operatorname{dim} f=3$.

Theorem 5.3. DBE $\left(\mathcal{Q}_{\text {inin }}\right)$ is probabilistic NP-hard.

Extensions. 1. The NP-hardness of Theorem 5.1 extends to all dimensions $n \geq 3$.

2. The proof of Theorem 5.1 can be extended from isotropic to anisotropic forms (that are used for identification and signatures). However, this requires a considerable amount of number theory and the Cohen-Lenstra heuristics for class numbers of real quadratic fields, see [10], [9]. The main problem is to show that all values $x^{2}+b y$ are covered by the anisotropic forms $x^{2}+b\left(y^{2}+z^{2}\right)$ for a few $b$.

3. At the end of this section we sketch how extend Theorem 5.3 from $\mathcal{Q}_{\text {inin }}$ to $\mathcal{Q}_{\text {ind }}$.

4. The problem $\mathbf{D B R}\left(\mathcal{Q}_{d f}\right)$ is $\mathbf{N P}$-complete in dimension $\geq 5$ even if the condition that 
$\left|x_{1}\right| \leq c$ is removed. This holds because the problem to decide whether $a x^{2}+b y=c$ has a solution $x, y \in \mathbb{N}$ is NP-hard [18], [9].

5.2 From SAT to squares. The following problem on binary Diophantine equations will be used as an intermediary problem for reductions.

\section{Modular Square Problem, MS}

GIVEN: $\quad a, b, c \in \mathbb{N}$ such that $b=p^{m+1} b^{\prime} ; a, p$ prime, $b^{\prime}$ squarefree, $p \geq 5$.

DECIDE: $\exists x \in \mathbb{Z}: x^{2} \equiv a \bmod b$ and $|x| \leq c$.

Let $\preccurlyeq r$ denote that there is a probabilistic, poly-time Karp reduction, and let 3SAT be the problem to decide satisfiability of Boolean forms in 3-CNF. Proposition 5.4 is similar to a result of ADLEMAN AND MANDERS [18].

Proposition 5.4. $3 S A T \preccurlyeq_{r}$ MS.

Proof. We transform a given Boolean form $\Phi$ in 3-CNF into an instance $(a, b, c)$ of MS that is solvable if and only if $\Phi$ is satisfiable. Let $\Phi$ contain each clause at most once, and let no clause of $\Phi$ contain a variable both complemented and uncomplemented. Let $\ell$ be the number of variables in $\Phi$. Choose an enumeration $\sigma_{1}, \ldots, \sigma_{m}$ of all such clauses in the variables $x_{1}, \ldots, x_{\ell}$ with exactly three literals such that the bijection $i \mapsto \sigma_{i}$ and its inverse are poly-time. We write $\sigma \in \Phi$ if $\sigma$ occurs in $\Phi$, and $x_{j} \in \sigma$ $\left(\bar{x}_{j} \in \sigma\right)$ if the $j$-th variable occurs uncomplemented (complemented) in clause $\sigma$. Let $n=2 m+\ell$. Let $r=\left(r_{1}, \ldots, r_{\ell}\right) \in\{0,1\}^{\ell}$ denote Boolean values for $x_{1}, \ldots, x_{\ell}$, where 1 corresponds to true and 0 corresponds to false. For a clause $\sigma$ and $r \in\{0,1\}^{\ell}$ define

$$
W(\sigma, r)=\sum_{i: x_{i} \in \sigma} r_{i}+\sum_{i: \bar{x}_{i} \in \sigma}\left(1-r_{i}\right) .
$$

Introducing new variables $y_{1}, \ldots, y_{m}$ we set for $k=1, \ldots, m$

$$
R_{k}:= \begin{cases}y_{k}-W\left(\sigma_{k}, r\right)+1 & \text { if } \sigma_{k} \in \Phi, \\ y_{k}-W\left(\sigma_{k}, r\right) & \text { if } \sigma_{k} \notin \Phi,\end{cases}
$$

Since $\Phi$ is in 3-CNF, we have $W\left(\sigma_{k}, r\right)=0$ if assignment $r$ does not satisfy clause $\sigma_{k}$, and $1 \leq W\left(\sigma_{k}, r\right) \leq 3$ otherwise. Hence the equations $R_{1}=\cdots=R_{m}=0$ have a solution $r \in\{0,1\}^{\ell}, y \in\{0,1,2,3\}^{m}$ if and only if $\Phi$ is satisfiable.

We select a prime $p \geq 5$. As $-3 \leq R_{k} \leq 4$ for all choices of $y_{k} \in\{0,1,2,3\}$, $r_{i} \in\{0,1\}$, the equations $R_{1}=\cdots=R_{m}=0$ holds if and only if $\sum_{k=1}^{m} R_{k} p^{k}=0$. The latter equation is equivalent to

$$
\sum_{k=1}^{m}\left(2 R_{k}\right) p^{k} \equiv 0 \bmod p^{m+1} .
$$

since $\left|\sum_{k=1}^{m} R_{k} p^{k}\right| \leq 4 \sum_{k=1}^{m} p^{k}<p^{m+1}$ holds for $p \geq 5$ and $p$ is odd.

We now add $\alpha_{0}+1$ to the left hand side of (5.1), where $\alpha_{0}$ is considered a variable taking values in $\{1,-1\}$. Hence (5.1) is solvable if and only if

$$
\alpha_{0}+1+\sum_{k=1}^{m}\left(2 R_{k}\right) p^{k} \equiv 0 \bmod p^{m+1} .
$$


for $y_{k}, r_{i}, \alpha_{0}$ ranging over their respective domains. Moreover, if (5.2) is solvable, then necessarily $\alpha_{0}=-1$ by reduction modulo $p$.

We express $y_{1}, \ldots, y_{m}$ and $r_{1}, \ldots, r_{\ell}$ in (5.2) by new variables $\alpha_{1}, \ldots, \alpha_{n}, n=$ $2 m+\ell$ ranging over $\{1,-1\}$, we set

$$
y_{k}:=\frac{1}{2}\left(\left(1-\alpha_{2 k-1}\right)+2\left(1-\alpha_{2 k}\right)\right), \quad r_{i}:=\frac{1}{2}\left(1-\alpha_{2 m+i}\right) .
$$

Exactly all combinations of $y_{k} \in\{0,1,2,3\}, r_{i} \in\{0,1\}$ are covered by $\alpha_{1}, \ldots, \alpha_{n}$. We can rewrite (5.2) via (5.3) into

$$
\sum_{j=0}^{n} c_{j} \alpha_{j} \equiv \tau \bmod p^{m+1}
$$

for some $c_{j}, \tau \in \mathbb{Z}$.We see that $\Phi$ is satisfiable if and only if (5.4) is solvable for $\alpha \in\{-1,1\}^{n+1}$. Note that $p \not \chi \tau$, as the constant part of (5.2) equals 1 .

We select a prime $p_{0}>4 \cdot p^{m+1}(n+1)$ and primes $p_{0}<p_{1}<\ldots<p_{n}$ that are of polynomial size in $p^{m}$. For $j=1, \ldots, n$ compute $\theta_{j}$ via the Chinese Remainder Theorem to be the smallest positive integer satisfying

$$
\theta_{j}\left\{\begin{array}{lll}
\equiv c_{j} & \bmod & p^{m+1} \\
\equiv 0 & \bmod & \prod_{i \neq j} p_{i}, \\
\neq \equiv 0 & \bmod & p_{j} .
\end{array}\right.
$$

We see that $\Phi$ is satisfiable if and only if

$$
\sum_{j=0}^{n} \theta_{j} \alpha_{j} \equiv \tau \bmod p^{m+1}
$$

is solvable for $\alpha \in\{ \pm 1\}^{n+1}$.

Lemma 5.5. Let $K:=\prod_{j=0}^{n} p_{j}$ and $c:=\sum_{j=0}^{n} \theta_{j}$. For $x \in \mathbb{Z},|x| \leq c$ the equation $c^{2} \equiv x^{2} \bmod K$ holds if and only if $x=\sum_{j=0}^{n} \alpha_{j} \theta_{j}$ for some $\alpha \in\{ \pm 1\}^{n+1}$.

Proof. Since $\theta_{j} \theta_{j^{\prime}} \equiv 0 \bmod K$ for $j \neq j^{\prime}$ each $x=\sum_{j=0}^{n} \alpha_{j} \theta_{j}$ satisfies $c^{2} \equiv x^{2} \bmod K$. Conversely, $c^{2} \equiv x^{2} \bmod K$ implies $0 \equiv(c-x)(c+x) \bmod K$, and thus either $p_{j} \mid c-x$ or $p_{j} \mid c+x$ holds for every $j=0, \ldots, n$. This disjunction is exclusive: if $p_{j} \mid c-x$ and $p_{j} \mid c+x$ then $p_{j} \mid 2 c$, and thus $p_{j} \mid c$. This contradicts the fact that $c \equiv \theta_{j} \not \equiv 0 \bmod p_{j}$.

Since $p_{j}$ divides exactly one of $c \pm x$ we define $\alpha_{j} \in\{ \pm 1\}$ such that $p_{j} \mid c-\alpha_{j} x$, hence $x \equiv \alpha_{j} c \bmod p_{j}$. Setting $x^{\prime}:=\sum_{i=0}^{n} \alpha_{i} \theta_{i}$ yields $x \equiv x^{\prime} \bmod p_{j}$ for all $j$, and thus $x \equiv x^{\prime} \bmod K$.

We see from $\left|x^{\prime}\right| \leq \sum_{j=0}^{n} \theta_{j}=c$ and $|x| \leq c$ that $\left|x-x^{\prime}\right| \leq 2 c$.

The choice of $p_{j}$ guarantees that $\frac{2 \cdot p^{m+1}}{p_{j}} \leq \frac{1}{2(n+1)}$, and thus

$$
\theta_{j}<2 \cdot p^{m+1} \prod_{\substack{i=0 \\ i \neq j}}^{n} p_{i}=\frac{2 \cdot p^{m+1} K}{p_{j}} \leq \frac{K}{2(n+1)} .
$$

Hence $c=\sum_{j=0}^{n} \theta_{j}<K / 2$. We see from $\left|x-x^{\prime}\right| \leq 2 c$ that $\left|x-x^{\prime}\right|<K$, and from $x \equiv x^{\prime} \bmod K$ that $x$ and $x^{\prime}$ coincide. This proves the lemma. 
Combining (5.5) with Lemma 5.5 we see that $\Phi$ is satisfiable if and only if there exists $x \in \mathbb{Z},|x| \leq c$ such that

$$
c^{2} \equiv x^{2} \bmod K, \quad x \equiv \tau \bmod p^{m+1} .
$$

These equations are equivalent to each of the following equations

$$
\begin{aligned}
c^{2} \equiv & x^{2} \bmod K, \tau^{2} \equiv x^{2} \bmod p^{m+1}\left(\mathbb{Z}_{p^{m+1}}^{*} \text { is cyclic }\right), \\
& p^{m+1}\left(c^{2}-x^{2}\right)+K\left(\tau^{2}-x^{2}\right) \equiv 0 \bmod p^{m+1} K, \\
& \left(p^{m+1}+K\right) x^{2} \equiv K \tau^{2}+p^{m+1} c^{2} \bmod p^{m+1} K .
\end{aligned}
$$

Since $p \nmid K$ the latter equation can be written as

$$
x^{2} \equiv a \bmod b
$$

$$
\text { for } b=p^{m+1} K \text { and } a \equiv\left(p^{m+1}+K\right)^{-1}\left(K \tau^{2}+p^{m+1} c^{2}\right) \bmod p^{m+1} K \text {. }
$$

Then (5.6) has a solution $x \in \mathbb{Z}$ with $|x| \leq c$ if and only if $\Phi$ is satisfiable. We can select a prime $a$ in the arithmetic progression (5.7) in random poly-time. Clearly $K$ is odd and squarefree, and the prime $a$ is coprime to $b$.

Then $(a, b, c)$ is a solvable instance of MS if and only if $\Phi$ is satisfiable.

5.3 Proofs of Theorems. Proof of Theorem 5.1. Let $\Phi$ be a boolean formula in 3CNF. The proof of Prop. 5.4 transforms $\Phi$ into an instance $(a, b, c)$ of MS, $a b$ odd, such that there exist $x, y \in \mathbb{Z}$ satisfying $x^{2}+b y=a,|x| \leq c$ if and only if $\Phi$ is satisfiable. We see that the form $f=2 x^{2}+2 b y z$ yields a solvable instance $f(x, y, z)=2 a$ of $\operatorname{DBR}\left(\mathcal{Q}_{\text {ind }}\right)$ if and only if $\Phi$ is satisfiable. For a primitive solution $(x, y, z)$ choose $z=1$. Note that $f$ is primitive since $b$ is odd. Moreover, $f$ is indefinite, isotropic and $\operatorname{det} f=-2 b^{2}$. This proves the claim.

Proof of Theorem 5.2. As in the proof of Prop. 5.4 we transform $\Phi$ into an instance $(a, b, c)$ of MS. Here we choose $a$ that satisfies equation (5.7) such that $a>c^{2}$. Then all integer solutions $x, y$ of $x^{2}+b y=a,|x| \leq c$ satisfy $y>0$. Lagrange's Four Square Theorem shows that $y \in \mathbb{N}$ can be represented as a sum of four squares. Therefore, we have transformed $\Phi$ into $(a, b, c) \in \mathbb{N}^{3}$ such that $x_{1}^{2}+b\left(x_{2}^{2}+\cdots+x_{5}^{2}\right)=a,\left|x_{1}\right| \leq c$ is solvable for $\left(x_{1}, \ldots, x_{5}\right) \in \mathbb{N}^{5}$ if and only if $\Phi$ is satisfiable. This proves the theorem.

Proof of Theorem 5.3. We transform instances $(a, b, c)$ of $\mathbf{M S}$ constructed in the proof of Prop. 5.4 into instances of $\mathbf{D B E}\left(\mathcal{Q}_{\text {inin }}\right)$, preserving solvability. We have that

$$
\left(\begin{array}{ccc}
a & b / 2 & x \\
b / 2 & 0 & 0 \\
x & 0 & 1
\end{array}\right)=\left(\begin{array}{ccc}
x & y & 1 \\
0 & 1 & 0 \\
1 & 0 & 0
\end{array}\right)\left(\begin{array}{ccc}
1 & 0 & 0 \\
0 & 0 & b / 2 \\
0 & b / 2 & 0
\end{array}\right)\left(\begin{array}{ccc}
x & 0 & 1 \\
y & 1 & 0 \\
1 & 0 & 0
\end{array}\right)
$$

for $a=x^{2}+b y$. Let $f=x^{2}+b y z$ and $f^{\prime}=a x^{2}+b x y+\gamma x z+z^{2}$ with $\gamma=*$, then $f=f^{\prime} T$ is solvable with $\left|t_{1,1}\right| \leq c$ if and only if $a=x^{2}+b y,|x| \leq c$ is solvable. 
NP-hardness of $\mathbf{D B E}\left(\mathcal{Q}_{\text {ind }}\right)$. We sketch how to extend the proof of Theorem 5.3 to reduce to complete forms in $\mathcal{Q}_{\text {ind }}$, for more details see [9]. We construct for $f=x^{2}+$ byz a list of forms $f_{1}, \ldots, f_{N}$ such that every solution $(x, y, z)$ of $a=x^{2}+b y z,|x| \leq c$ provides a first column of some $S \in \mathrm{GL}_{3}(\mathbb{Z})$ satisfying $f S=f_{j}$ for some $f_{j}$. Hence $(a, b, c)$ is solvable for MS if and only if $\left(f, f_{j}, c\right)$ is for some $1 \leq j \leq N$ solvable for DBE. This proves the desired reduction.

We construct the $f_{j}$ to represent all orbits of primitive representations of $a$ by $f$ under $\mathcal{O}(f)$. ZHURAVLEV [28, sec. 1.2] classifies these orbits in terms of modular matrix equations. We construct the $f_{j}$ by solving these equations. First we determine the genus of $f_{j}$, i.e., the $f_{j}$ up to simultaneous equivalence over the reals $\mathbb{R}$ and over all rings of $p$-adic integers. Then we construct the actual $f_{j}$ by a constructive version of the classical proof of the existence of genera [3, sec. 9.5]. According to MS, the integer $a$ is prime, and this ensures that $N=O(1)$. Moreover, the construction is poly-time per entry $f_{j}$, given the factorization of $a b$.

\section{Solving quadratic equations using an isotropic vector}

Isotropic forms $f_{A}$ are universal over any field $\mathbb{F}$, i.e., for $A \in \mathbb{F}^{n \times n}$ the equation $f_{A}(\mathbf{x})=m$ is solvable for all $m \in \mathbb{F}$ if it is solvable for $m=0, \mathbf{x} \neq 0$, [3, chap. 2.2]. We show that $f_{A}(\mathbf{x})=m$ can easily be solved for all $m \in \mathbb{Z}$ over the integers if an isotropic vector is given and $\operatorname{det} A$ is odd and squarefree. Theorem 6.1 transforms an isotropic form $f_{A}$ with odd, squarefree determinant $d=\operatorname{det} A$ into the form $2 x y-d z^{2}$ and Theorem 6.2 solves the equation $2 x y-d z^{2}=m$. Importantly, Simon [26], [27] has shown that an isotropic vector can be found in poly-time for any isotropic form $f$ given the factorization of $\operatorname{det} f$.

Theorem 6.1. Let $f_{A}$ be an isotropic, ternary form and let $d:=\operatorname{det} A$ be odd and squarefree. Given an isotropic vector $\left(x^{\prime}, y^{\prime}, z^{\prime}\right)$ the form $f_{A}$ can be transformed in poly-time into the equivalent form $2 x y-d z^{2}$.

Proof. Make $\left(x^{\prime}, y^{\prime}, z^{\prime}\right)$ primitive by dividing it by $\operatorname{gcd}\left(x^{\prime}, y^{\prime}, z^{\prime}\right)$. Compute some $S \in$ $\mathrm{GL}_{3}(\mathbb{Z})$ with first column vector $\left(x^{\prime}, y^{\prime}, z^{\prime}\right)^{t}=S(1,0,0)^{t}$ as follows:

Compute $g:=\operatorname{gcd}\left(y^{\prime}, z^{\prime}\right)$ and $S_{1}, S_{2} \in \mathrm{GL}_{3}(\mathbb{Z})$ such that $S_{1}\left(x^{\prime}, y^{\prime}, z^{\prime}\right)^{t}=\left(x^{\prime}, g, 0\right)^{t}$, $S_{2}\left(x^{\prime}, g, 0\right)^{t}=(1,0,0)^{t}$. Set $S^{-1}:=S_{2} S_{1}$ since $S_{2} S_{1}\left(x^{\prime}, y^{\prime}, z^{\prime}\right)^{t}=S_{2}\left(x^{\prime}, g, 0\right)^{t}=$ $(1,0,0)^{t}$. To compute $S_{1}$ solve $y^{\prime} a+z^{\prime} b=g$ by the extended gcd-algorithm. Then $S_{1}^{\prime}:=\left(\begin{array}{cc}a & b \\ z^{\prime} / g & -y^{\prime} / g\end{array}\right) \in \mathrm{GL}_{2}(\mathbb{Z})$ satisfies $S_{1}^{\prime}\left(y^{\prime}, z^{\prime}\right)^{t}=(g, 0)^{t}$. Extend $S_{1}^{\prime}$ to $S_{1} \in$ $\mathrm{GL}_{3}(\mathbb{Z})$ by adding the first row $(1,0,0)$ and first column $(1,0,0)^{t}$. Hence $S_{1}\left(x^{\prime}, y^{\prime}, z^{\prime}\right)^{t}$ $=\left(x^{\prime}, g, 0\right)^{t}$. Compute $S_{2}$ accordingly by solving $x^{\prime} a+g b=1=\operatorname{gcd}\left(x^{\prime}, g\right)$.

Given $S$ set $A^{\prime}=\left(a_{i, j}^{\prime}\right)_{1 \leq i, j \leq 3}:=S^{t} A S$. Then $a_{1,1}^{\prime}=0$ as $\left(x^{\prime}, y^{\prime}, z^{\prime}\right)$ is isotropic.

Compute via the extended gcd-algorithm some $V \in \mathrm{GL}_{2}(\mathbb{Z})$ such that $\left(a_{1,2}^{\prime}, a_{1,3}^{\prime}\right) V=$ $(r, 0)$ holds for $r:=\operatorname{gcd}\left(a_{1,2}^{\prime}, a_{1,3}^{\prime}\right)$. This yields integers $g, t, h$ such that 


$$
\left(\begin{array}{ll}
1 & \\
& V^{t}
\end{array}\right) A^{\prime}\left(\begin{array}{ll}
1 & \\
& V
\end{array}\right)=\left(\begin{array}{lll}
0 & r & 0 \\
r & g & t \\
0 & t & h
\end{array}\right) .
$$

As $d=-r^{2} h$ and $d$ is squarefree we have $r= \pm 1$. So let $r=1, h=-d$, and thus

$$
\left(\begin{array}{ccc}
1 & & \\
-\lfloor g / 2\rfloor & 1 & \\
-t & & 1
\end{array}\right)\left(\begin{array}{lll}
1 & \\
1 & g & t \\
& t & h
\end{array}\right)\left(\begin{array}{ccc}
1 & -\lfloor g / 2\rfloor & -t \\
& 1 & \\
& & 1
\end{array}\right)=\left(\begin{array}{ccc}
1 & \\
1 & e & 0 \\
& 0 & -d
\end{array}\right)
$$

where $e=g-2\lfloor g / 2\rfloor \in\{0,1\}$. So far we have transformed $f_{A}$ into $2 x y+e y^{2}-d z^{2}$. We are finished if $e=0$.

Case e $=1$. We transform $2 x y+y^{2}-d z^{2}$ into $2 \bar{x} y-d \bar{z}^{2}$. As $d$ is odd let $-d=1+2 k$. Transforming $2 x y+y^{2}-d z^{2}$ via $x:=\bar{x}+k y-d z$ yields

$$
2 \bar{x} y+2 k y^{2}+y^{2}-2 d y z-d z^{2}=2 \bar{x} y-d y^{2}-2 d y z-d z^{2}=2 \bar{x} y-d(y+z)^{2} .
$$

Setting $\bar{z}:=y+z$ yields $2 \bar{x} y-d \bar{z}^{2}$.

Theorem 6.2. Let $d \in \mathbb{Z}$ be odd. Then the equation $2 x y-d z^{2}=m$ is solved by any $z$ satisfying $z=m \bmod 2$ and $x:=\left(m+d z^{2}\right) /(2 y)$ where $y$ is an arbitrary integer divisor of $\left(m+d z^{2}\right) / 2$.

Proof. For odd $d$ we have that $m+d z^{2}$ is even if and only if $z=m \bmod 2$. Hence $\left(m+d z^{2}\right) / 2$ is integer and the claimed $(x, y, z)$ is a solution.

Extensions. Ternary isotropic forms $f_{A}$ of odd, squarefree det $A$ are equivalent if and only if they coincide in $\operatorname{det} A$. Moreover, CEP for such $f_{A}$ can be solved in poly-time by Theorem 6.1. In particular, such $f_{A}$ is equivalent to $2 x y-\operatorname{det} A z^{2}$ by Theorem 6.1. However, $f_{A}$ can be inequivalent to $2 x y-\operatorname{det} A z^{2}$ for even $\operatorname{det} A$. For example, the forms $a x^{2}+b y^{2}-c z^{2}$ and $2 x y+a b c z^{2}$ can be inequivalent for even $a b c$ because only the second form is a multiple of 2 unless $a, b, c$ are all even.

The equation $2 x y-d z^{2}=m$ is unsolvable for even $d$ and odd $m$. However, all solvable instances of $f_{A}(\mathbf{x})=m$ for even, squarefree $d=\operatorname{det} A$ can easily be solved given an isotropic vector.

Even when $d=\operatorname{det} A$ is not squarefree the equation $f_{A}(\mathbf{x})=m$ can in practice be solved by the method of Theorems 6.1, 6.2. It is unlikely that a large squarefactor $r^{2} \neq 1$ shows up in the algorithm of Theorem 6.1.

The set of all solutions. We get all solutions $(x, y, z) \in \mathbb{Z}^{3}$ of $2 x y-d z^{2}=m$ by extending the solutions of Theorem 6.2 in that we allow to permute $x$ and $y$ and to change the signs of $x, y, z$. In fact, we easily get all solutions of $2 x y-d z^{2}=0$ given the factorization of $d$.

Moreover, when we replace $\mathbb{Z}$ by a finite field, a finite ring or the field of real numbers then solving the equation $f_{A}(\mathbf{x})=m$ is relatively easy for $m \neq 0$. Solutions over the ring $\mathbb{Z}_{N}, N$ composite, can be found using Pollard's algorithm [24]. 


\section{Characterization of isotropic and anisotropic indefinite forms}

For fixed $n$, every $n$-ary form $f$ over $\mathbb{Z}$ can be transformed in poly-time into a diagonal form $f T=\frac{1}{a_{0}} f^{\prime}=\frac{1}{a_{0}} \sum_{i=1}^{n} a_{i} x_{i}^{2}$, where $a_{0}, \ldots, a_{n} \in \mathbb{Z}$ and $T \in \mathbb{Q}^{n \times n}$, $\operatorname{det} T=1$. Then $f$ is isotropic if and only if $f^{\prime}$ is isotropic. Next we characterize isotropic diagonal forms. The form $a x^{2}+b y^{2}-c z^{2}$ is isotropic if and only if the Legendre equation

$$
a x^{2}+b y^{2}=c z^{2}
$$

is solvable. The equation (7.1) is in normal form if $a, b, c \in \mathbb{N}$ are positive, squarefree and pairwise coprime. Let $Q R_{a}$ denote the set of quadratic residues modulo $a$.

Theorem 7.1. (see [17], [4]) In normal form the equation (7.1) has a non-zero solution if and only if $b c \in Q R_{a}, a c \in Q R_{b}$ and $-a b \in Q R_{c}$, and solving (7.1) for given $a, b, c$ is poly-time equivalent to each of the following problems :

1. solve $\alpha^{2}=b c \bmod a, \beta^{2}=a c \bmod b$ and $\gamma^{2}=-a b \bmod c$,

2. solve equation (7.1) for a non-zero $(x, y, z) \in \mathbb{Z}^{3}$ such that $x^{2}+y^{2}+z^{2} \leq 2 a b c$.

Theorem 7.2. [3, chap. 4.1, 4.2, lem. 2.6] An indefinite form $f=\sum_{i=1}^{4} a_{i} x_{i}^{2}$ with $a_{i} \in \mathbb{Z}$ is anisotropic if and only if there exist a prime $p$ and $k \in \mathbb{N}$ such that $p^{2 k} \mid d$, $p^{2 k+1} \backslash d$ for $d:=a_{1} a_{2} a_{3} a_{4}$ and $\prod_{i<j}\left(a_{i}, a_{j}\right)_{p}=(-1)^{p \bmod 2}$.

The Hilbert Norm Residue Symbol $\left(a_{i}, a_{j}\right)_{p} \in\{ \pm 1\}$ equals 1 if and only if $a_{i} x^{2}+$ $a_{j} y^{2}-z^{2}$ is isotropic over $\mathbb{Q}_{p}$, the field of $p$-adic numbers. In particular $\left(a_{i}, a_{j}\right)_{p}$ is poly-time, and anisotropy of $f$ is poly-time given the factorization of $\operatorname{det} f$ [3, chap. 3.2]. For $n \geq 5$ every indefinite $n$-ary form $f$ is isotropic [19]. Anisotropic quaternary forms $f$ have a square-factor dividing $\operatorname{det} f$.

\section{References}

[1] M. Ajtai and Cynthia Dwork, A public-key cryptosystem with worst-caselaverage-case equivalence. Proceedings STOC 1997, pp. 284-293, Association for Computing Machinery, New York, 1997.

[2] J. Buchmann and U. Vollmer, Binary quadratic forms. An algorithmic approach, Algorithms and Computation in Mathematics 20. Springer-Verlag, Berlin, 2007.

[3] J. W. S. Cassels, Rational quadratic forms, L.M.S. Monographs 13. Academic Press, New York, 1978.

[4] T. Cochrane and P. Mitchell, Small solutions of the Legendre equation, Journal of Number Theory 70 (1998), pp. 62-66.

[5] H. Cohen, A course in computational algebraic number theory, Graduate Texts in Mathematics 138. Springer-Verlag, Berlin, 1993.

[6] R. Dietmann, Small solutions of quadratic Diophantine equations, Proceedings of the London Mathematical Society, III. Ser. 86 (2003), pp. 545-582.

[7] C. F. Gauß, Untersuchungen über höhere Arithmetik (Disquisitiones Arithmeticae). SpringerVerlag, Berlin, 1889, second edition: Chelsea Publ. New York, 1965, reprinted 1981.

[8] O. Goldreich, Shafi Goldwasser, and S. Halevi, Public-key cryptosystems from lattice reduction problems. Proceedings Advances in Cryptology - CRYPTO '97, Lecture Notes in Computer Science 1294, pp. 112-131. Springer-Verlag, Berlin, 1997. 
[9] R. J. Hartung, Computational Problems of Quadratic Forms: Complexity and Cryptographic Perspectives, Ph. D. thesis, Goethe-Universität Frankfurt a. M., 2008, http://nbn.resolving.de/urn/resolver.pl?urn:nbn:de:hebis:30-54444.

[10] R. J. Hartung and C.-P. Schnorr, Public key identification based on the equivalence of quadratic forms. Proceedings MFCS 2007, Lecture Notes in Computer Science 4708, pp. 333-345. Springer-Verlag, Berlin, 2007, http://www.mi.informatik.uni-frankfurt.de.

[11] J. Hoffstein, N. Howgrave-Graham, J. Pipher, J. H. Silverman, and W. Whyte, NTRUSign: Digital signatures using the NTRU lattice. Proceedings Topics in cryptology - CT-RSA 2003, Lecture Notes in Computer Science 2612, pp. 122-140. Springer-Verlag, Berlin, 2003.

[12] J. Hoffstein, J. Pipher, and J. H. Silverman, NTRU: A ring-based public key cryptosystem. Proceedings 3rd international symposium ANTS-III, 1998, Lecture Notes in Computer Science 1423, pp. 267-288. Springer-Verlag, Berlin, 1998.

[13] G. Ivanyos and Á. Szánto, Lattice basis reduction for indefinite forms and an application, Journal on Discrete Mathematics 153 (1996), pp. 177-188.

[14] H. W. Lenstra jun., A. K. Lenstra, and L. Lovász, Factoring polynomials with rational coefficients, Mathematische Annalen 261 (1982), pp. 515-534.

[15] S. Khot, Hardness of approximating the shortest vector problem in lattices, Journal of the ACM 52 (2005), pp. 789-808.

[16] J. C. Lagarias, Worst-case complexity bounds for algorithms in the theory of integral quadratic forms, Journal of Algorithms 1 (1980), pp. 142-186.

[17] A. M. Legendre, Recherche d'analyse indéterminée, Histoire de l'Académie Royale des Sciences 1788 (1785), pp. 465-559.

[18] K. L. Manders and L. M. Adleman, NP-complete decision problems for binary quadratics, Journal of Computer and System Sciences 16 (1978), pp. 168-184.

[19] A. Meyer, Zur Theorie der indefiniten ternären quadratischen Formen, Journal für Mathematik 108 (1891), pp. 125-139.

[20] D. Micciancio and Shafi Goldwasser, Complexity of lattice problems: A cryptographic perspective, The Kluwer International Series in Engineering and Computer Science 671. Kluwer, Boston, 2002.

[21] P. Q. Nguyen and D. Stehlé, Floating-point LLL revisited. Proceedings Advances in Cryptology - EUROCRYPT 2005, Lecture Notes in Computer Science 3494, pp. 215-233. SpringerVerlag, Berlin, 2005.

[22] W. Plesken and M. Pohst, Constructing integral lattices with prescribed minimum. I, Mathematics of Computation 45 (1985), pp. 209-221.

[23] W. Plesken and B. Souvignier, Computing isometries of lattices, Mathematics of Computation 45 (1985), pp. 209-221.

[24] J. M. Pollard and C.-P. Schnorr, An efficient solution of the congruence $x^{2}+k y^{2}=m(\bmod n)$, IEEE Transactions on Information Theory 33 (1987), pp. 702-709.

[25] C.-P. Schnorr, Progress on LLL and lattice reduction. Proceedings LLL+25, Caen, France, June 29-July 1, 2007, Final version to appear; http://www.mi.informatik.uni-frankfurt.de.

[26] D. Simon, Quadratic equations in dimensions 4, 5 and more, 2005, preprint Uni. Caen: http://www.math.unicaen.fr/s̃imon/.

[27] __ Solving quadratic equations using reduced unimodular quadratic forms, Mathematics of Computation 74 (2005), pp. 1531-1543.

[28] V. G. Zhuravlev, Representation of a Form by the Genus of Quadratic Forms, St. Petersburg Mathematical Journal 8 (1997), pp. 15-84. 


\section{Received}

\section{Author information}

Rupert J. Hartung, Johann Wolfgang Goethe Universität Frankfurt a. M.

Postfach 11 19 32; Fach 238

60054 Frankfurt a. M., Germany.

Email: hartung@math.uni-frankfurt.de

Claus-Peter Schnorr, Johann Wolfgang Goethe Universität Frankfurt a. M.

Postfach 11 19 32; Fach 238

60054 Frankfurt a. M., Germany.

Email: schnorr@mi.informatik.uni-frankfurt.de 